\title{
A Combinatorial Analysis of Distance Reliability in Star Network
}

\author{
Xiaolong $\mathrm{Wu}$, Shahram Latifi, and Yingtao Jiang \\ Department of Electrical and Computer Engineering \\ University of Nevada, Las Vegas, NV 89154, USA \\ \{xiaolong, latifi,yingtao\}@egr.unlv.edu
}

\begin{abstract}
This paper addresses a constrained two-terminal reliability measure referred to as Distance Reliability (DR) between the source node $u$ and the destination node $I$ with the shortest distance, in an n-dimensional star network, $S_{n}$. The shortest distance restriction guarantees the optimal communication delay between processors and high link/node utilization across the network. This paper uses a combinatorial approach by limiting the number of node, link and nodellink failures. For each failure model, two different cases depending on the relative positions of $u$ and $I$, are analyzed to compute DR. Furthermore, DR for the antipodal communication, where every node must communicate with its antipode, is investigated as a special case. For this case, lower bound on DR of those disjoint paths is also derived.
\end{abstract}

\section{Introduction}

Star networks have been known as a viable candidate for interconnecting a large number of processors [1][2]. The suitability of a star architecture (like any parallel architecture) is evaluated by analyzing its performance and reliability aspects. Previous researches have investigated star networks using performance metrics such as number of nodes and links, connectivity, diameter, fault diameter, etc [3][6][8][10][11]. However, little attention has been paid to the reliability issues. The reliability or availability prediction of a star architecture is quite essential since stars have the potential of use in critical applications.

Because of high similarity between hypercube and star network [5][7], star network is highly robust. It has been proved that the connectivity among nodes in this topology can be preserved despite a substantial number of failures (in terms of node, link or node/link failures). This fact motivated us to look beyond the concept of connectivity, and demand more of this topology in terms of efficient communication. Hence, we want a given pair of nodes not only to be connected but to be apart by the optimal distance (i.e. the shortest distance between the source node $u$ and the destination node $I$ ). This idea consequently arouses a distance constrained reliability parameter which serves as a useful assessment to determine the communication delay, link-node utilization, and robustness. We define Distance
Reliability $(D R)$ as the probability of having an operational path with the optimal distance between two given nodes $u$ and $I$. We propose a combinatorial method to evaluate $D R$ for any $(u, I)$-containers in a $S_{n}$, under the node failure, link failure and node/link failure models, respectively. Meanwhile, $D R$ for the antipodal communication will be discussed as a special case here.

The paper is organized as follows. In Section 2, we introduce basic properties of star network. In Section 3, a combinatorial approach is proposed to derive $D R$ under the node failure model. Section 4 continues to discuss $D R$ under the link failure model. And the combined node and link failure model is used to derive $D R$ in Section 5. Section 6 concludes the paper.

\section{Background}

A star network of dimension $n, S_{n}$ is defined as a Calyley Graph $G=(V, E)$ where $V$ is the set of $n$ !nodes, and $E$ is the set of $(n-1) n ! / 2$ links. The nodes are assigned labels each of which is a distinct permutation on $n$ symbols (we use symbols $1,2, \ldots, n)$. Two nodes are joined with a link labeled $i$ if and only if the label of one can be obtained by swapping the first digit (leftmost) and the $i^{\text {th }}$ digit, where $1<i \leq n$. For example, in a $S_{4}$ containing 24 nodes, two nodes 1234 and 3214 are neighbors and joined via a link labeled 3.

\subsection{Routing in star network}

Since the star network is node symmetric (i.e. network looks the same from every node's viewpoint), for routing between two nodes, the destination node is commonly assumed to have the identity permutation $I=[12 \ldots n]$ as its label. Having the label of the destination node fixed to $I$, one way to specify the source node is to exploit the fact that the label, $u$, is simply a permutation on the digits of $I$. Routing between two given nodes is accomplished based on following two rules [1].

i) If 1 is the leftmost digit, move it to any position not occupied by the correct digit, and

ii) If $i$ is the leftmost digit $(1<i \leq n)$, move it to its correct position.

Any permutation can be viewed as a set of cycles, i.e. cyclically order sets of digits with the property that each 
digit's desired position is that occupied by next digit in the set. For example, the source node labeled as [426513] in a $S_{6}$, can also be identified by the cyclic representation of its label, i.e. (145)(2)(36). Note that, any digit already in the correct position appears in a cycle of length of 1, or a 1cycle. From above, it follows that in the representation of a given permutation by a set of cycles, cycles can appear in any order; furthermore, within each cycle, any cyclic shift of the sequence of digits can be used without affecting the destination node. For instance, in a $S_{5}$, representations: (123)(45), (45)(123), and (312)(45) all specify the same source node labeled as [23154].

\subsection{Disjoint paths in star network}

The maximum number of node-disjoint paths of the shortest length between a given pair of nodes in $S_{n}$ has been derived in [5] and is known to be " $n-1$ ". The notation $\pi(i)$ is used to refer to the $i^{\text {th }}$ digit of the label of $u$. If the label of $u$, expressed in its cyclic form, has $c$ cycles of length at least 2 and $m$ misplaced digits $(m \leq n)$, then it follows:

i) If $\pi(1)=1$, there are $n-1$ parallel node-disjoint paths between $u$ and $I$ as follows:

a) $m$ paths of the shortest length $c+m$;

b) $n-m-1$ paths of length $c+m+2$.

ii) If $\pi(1) \neq 1$, there are $n-1$ parallel node-disjoint paths between $u$ and $I$ as follows:

a) $c$ paths of the shortest length $c+m-2$;

b) $m-c-1$ paths of length $c+m$, and

c) $n-m$ paths of length $c+m+2$.

For the purpose of simplicity, we use $r$ to denote the shortest distance between $u$ and $I$ in the rest of this paper.

\subsection{Antipode of a node in star network}

In a network, the farthest node(s) from a given node along the shortest path is called the node's antipode(s) [9]. The antipode of a node is apart from it by $d_{n}$ (diameter of star network) and can be specified by a maximum permutation $\left(\delta_{\max }\right)$. This scenario is explained in [9] as follows:

i) When $n$ is odd, all cycles of permutations $\delta_{\max }$ must be of length 2 with digit 1 in place. Then $\delta_{\text {max }}=(1)\left(i_{2} i_{3}\right)\left(i_{4} i_{5}\right) \ldots\left(i_{n-1} i_{n}\right)$, where $1<i_{2}, i_{3}, \ldots, i_{n} \leq \mathrm{n}$.

Here $m=n-1, c=(n-1) / 2$.

ii) When $n$ is even, there are two possibilities. First, there is one cycle of length 3 and the rest of cycles have the length 2 (digit 1 must be in place), $\delta_{\max }=(1)\left(i_{2} i_{3} i_{4}\right)\left(i_{5} i_{6}\right) \ldots\left(i_{n-1} i_{n}\right)$, where $1<i_{2}, i_{3}, \ldots, i_{n} \leq \mathrm{n}$.

Here, $\quad m=n-1$ and $c=(n-2) / 2$. Second, there are $n / 2$ 2-cycles with one cycle containing digit 1 , $\delta_{\max }=\left(i_{1} i_{2}\right)\left(i_{3} i_{4}\right)\left(i_{5} i_{6}\right) \ldots\left(i_{n-1} i_{n}\right)$, where $1<i_{2}, i_{3}, \ldots, i_{n} \leq \mathrm{n}$.

As an example, in a 5 -star, one of the antipodes of a given node can be specified by the following permutation:

$$
\delta_{\text {max }}=(1)(23)(45)
$$

And for a 6 -star, there are two possibilities to specify the antipodes. For example, $\delta_{\max }$ can be either of:

$$
\text { (1)(234)(56) or (12)(34)(56) }
$$

\section{Node failure model}

Let $F$ be the set of faulty nodes with $|F|$ denoted as the number of the faulty nodes. Here, we only focus on the case with at most $|F|$ node failures. Links are assumed to be perfect under this model. The objective is to find $D R$, i.e. probability of having at least one operational path of the shortest distance between $u$ and $I$ at the presence of node failures. A path is operational if it passes through fault-free intermediary nodes. From Section 2.2, the shortest distance of paths between $u$ and $I$ is either $c+m$ or $c+m-2$.

\subsection{Case I: $\pi(1)=1$}

There are $m$ optimal node-disjoint paths of the shortest distance $c+m$, and $n-m-1$ non-optimal paths of distance $c+m+2$ between $u$ and $I \quad(m<n, c \leq\lfloor(n-1) / 2\rfloor$, and $r=c+m$.

Definition 1. The union of all $m$ optimal paths existing between $u$ and $I$ is referred to as a $(u, I)$-container. Total distinct nodes existing in this container is $k=m(r-1)+2$.

Lemma 1. If $|F|<m$, there will be at least one optimal operational path between $u$ and $I$.

Proof: In a star network there exist $m$ node-disjoint parallel paths of the shortest distance $r$ between $u$ and $I$. Each faulty node can at most belong to one path and sincel $F \mathrm{~K} m$, there will be at least one operational optimal path existing between $u$ and $I$.

Corollary 1. A star network is distance reliable for pairs of $u$ and $I$, if $|F|<m$. Therefore it follows:

$$
D R=1,|F|<m
$$

Theorem 1 In a container consisting of $m$ optimal paths between two given nodes $u$ and $I$, all links incident on $u$ (or I) are unique. .

Proof: For any two optimal paths between $u$ and $I$, which have two given unique links " $i$ " and " $j$ " incident on $u$, assume there is the same link " $k$ " incident on $I$ for these two optimal paths. Based on the basic permutation of star networks, the last node in these paths before reaching $I$ will be the same. These two paths having two links " $i$ " and " $j$ " incident on $u$ are not node-disjoint anymore. This is in 
contradiction with the conclusion in [5] stating that there are $m$ optimal node-disjoint paths between $u$ and $I$. Q.E.D.

Theorem 1 can be further clarified by the following example. There are four optimal node-disjoint paths in one of the containers between [132546] and I. In Fig. 1, for the optimal path incident on $u$ with link " 3 ", there is a unique link " 5 " incident on $I$ such that the optimal path 3-2-3-5-4-5 can be constructed in this $(u, I)$-container. Other links in this $(u, I)$-container follow the same pattern.

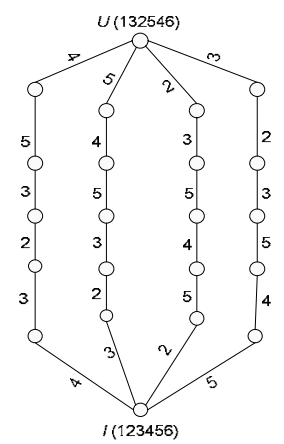

Figure 1. Four node-disjoint paths between two nodes.

Now we investigate $D R$ when $|F|=m$. If all faults happen to be in one of the optimal paths, one optimal faultfree path between $u$ and $I$ can be guaranteed. Here we consider the worst scenario: $m$ faults distributions will destroy all optimal $m$ paths. This can only happen when all $m$ faults are neighbors of either $u$ or $I$. The conditional probability associated with this event is:

$$
\begin{aligned}
& \operatorname{Pr}(\text { all r-paths destroyed }|| F|=m|= \\
& 2\left\{\left(\begin{array}{c}
m \\
m
\end{array}\right)+\left(\begin{array}{c}
m \\
m-1
\end{array}\right)+\ldots+\left(\begin{array}{c}
m \\
0
\end{array}\right)\right\} /\left(\begin{array}{c}
k-2 \\
m
\end{array}\right)=2^{m+1} /\left(\begin{array}{c}
k-2 \\
m
\end{array}\right)
\end{aligned}
$$

Above equation accounts for the $2^{m+1}$ mutually-exclusive distributions that destroy $m$ optimal paths. Subtraction of the probability of the occurrence of these events from 1 will naturally give the probability of having at least one operational $r$-path in the container and thus the $D R$. Two of them are shown in Fig. 2. In Fig. 2(a), $u$ has $m$ faulty neighbors and all neighbors of $I$ are fault-free; Fig. 2(b) is a general case where $u$ has $i$ arbitrary faulty neighbors and $m$ $i$ fault-free neighbors, and $I$ has $m-i$ faulty neighbors (none of them is in the path having any faulty neighbors of $u$ ) and $i$ fault-free neighbors (each of them is in the path having one of faulty neighbors of $u$ ).

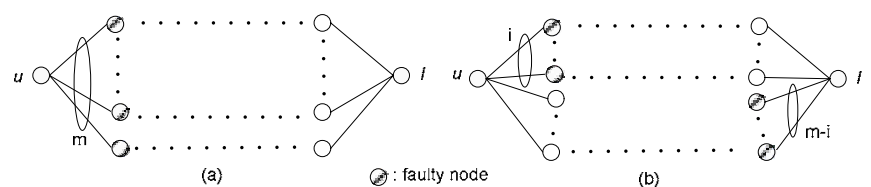

Figure 2. Two fault distributions.
In reality the likelihood of having all $r$-paths failed due to node failures is very small. The probability of having at least one optimal path when $|F|=m$ are presented in Table 1 . In our experiments, we only consider the case with maximal $m$ and $c$. The first column is the dimension of star network, the second and third ones are values for $m$ and $c$, the fourth one is the shortest distance, the fifth one is total nodes in the container except $u$ and $I$, and the last column is the probability of existing at least one optimal path between $u$ and $I$, separately. For example, the existence of at least one optimal path between $u$ and $I$ can be assured when the shortest distance between two given nodes is larger than 7 . This is can be visualized in Fig. 3 .

Table 1. Prob. of destroying all $r$-paths when $|F|=m$.

\begin{tabular}{|c|c|c|c|c|c|}
\hline$n$ & $\begin{array}{c}\text { max. } \\
m\end{array}$ & $\begin{array}{c}\text { max. } \\
c\end{array}$ & $r$ & $\begin{array}{c}\text { \# of nodes } \\
\text { in container }\end{array}$ & $\begin{array}{c}\text { Prob. having at least } \\
\text { one optimal path }\end{array}$ \\
\hline 4 & 3 & 1 & 4 & 9 & 0.80952381 \\
\hline 5 & 4 & 2 & 6 & 20 & 0.993395253 \\
\hline 6 & 5 & 2 & 7 & 30 & 0.999550896 \\
\hline 7 & 6 & 3 & 9 & 48 & 0.999989569 \\
\hline 8 & 7 & 3 & 10 & 63 & 0.999999537 \\
\hline 9 & 8 & 4 & 12 & 88 & 0.999999992 \\
\hline 10 & 9 & 4 & 13 & 108 & 1 \\
\hline
\end{tabular}

Table 2. Prob. of destroying all $r$-paths when $|F|=c$.

\begin{tabular}{|c|c|c|c|c|c|}
\hline$n$ & $\begin{array}{c}\max . \\
m\end{array}$ & $\begin{array}{c}\max . \\
c\end{array}$ & $r$ & $\begin{array}{c}\text { \# of nodes } \\
\text { in container }\end{array}$ & $\begin{array}{c}\text { Prob. having at least } \\
\text { one optimal path }\end{array}$ \\
\hline 4 & 4 & 2 & 4 & 10 & 0.822222222 \\
\hline 5 & 5 & 2 & 5 & 12 & 0.878787879 \\
\hline 6 & 6 & 3 & 7 & 24 & 0.992094862 \\
\hline 7 & 7 & 3 & 8 & 27 & 0.994529915 \\
\hline 8 & 8 & 4 & 10 & 44 & 0.999764274 \\
\hline 9 & 9 & 4 & 11 & 48 & 0.999835543 \\
\hline 10 & 10 & 5 & 13 & 70 & 0.999994712 \\
\hline
\end{tabular}

\subsection{Case II: $\pi(1) \neq 1$}

The container under this case has $c$ optimal paths of the shortest distance $c+m-2, m-c-1$ paths of distance $c+m$, and $n-m$ paths of distance $c+m+2$ between $u$ and $I$ $\left(m \leq n, c \leq\left\lfloor\frac{n}{2}\right\rfloor\right.$, and $\left.r=c+m-2\right) \quad$ with $\quad k=c(r-1)+2$ distinct nodes in one $(u, I)$-container.

There will be at least one operational path between $u$ and $I$ if $|F|<c$. Further, since nodes are equally likely to fail, it is possible to have one operational path between $u$ and $I$ even when $|F|<c$.

Now we investigate $D R$ when $|F|=c$. The conditional probability associated with this event is:

$$
\begin{aligned}
& \operatorname{Pr}(\text { all r-paths destroyed }|| F|=c|= \\
& 2\left\{\left(\begin{array}{l}
c \\
c
\end{array}\right)+\left(\begin{array}{c}
c \\
c-1
\end{array}\right)+\ldots+\left(\begin{array}{l}
c \\
0
\end{array}\right)\right\} /\left(\begin{array}{c}
k-2 \\
c
\end{array}\right)=2^{c+1} /\left(\begin{array}{c}
k-2 \\
c
\end{array}\right)(3)
\end{aligned}
$$


These $2^{c+1}$ mutually-exclusive distributions are similar to Case I except different number of node-disjoint paths of the shortest distance. The probability of existing at least one optimal path with the increase of the shortest distance between two given nodes when $|F|=c$ is shown in Table 2 . From Fig. 3, it can be concluded that the existence of at least one optimal path between $u$ and $I$ can be assured when the shortest distance is larger than 10.

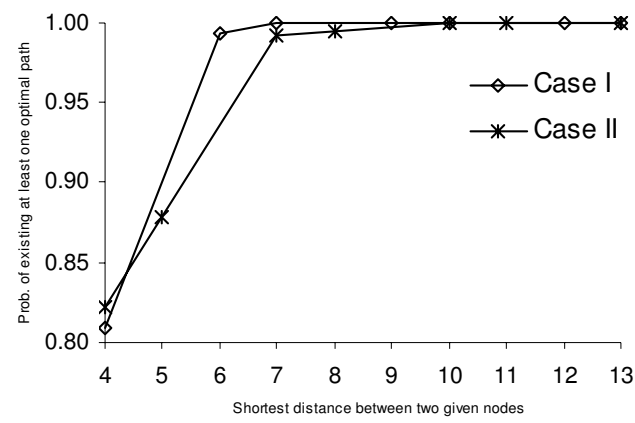

Fig. 3. Prob. of existence of at least one optimal path.

\subsection{Special case: antipode reliability}

\section{a. Stochastic model}

Determinations of $D R$ when $|F|$ is greater than the number of node-disjoint paths, is difficult due to the numerous possible distributions. Under this circumstance, and for a given $|F|$ obtained from the network reliability data and its mission time, we can develop reliability expression based on a stochastic model as follows.

In the stochastic graph model $G(V, E)$ for the star network $S_{n}$, the following assumptions are made:

i) Source and destination nodes are always fault-free;

ii) The operational probability of all nodes (links) are the same and is equal to $p_{n}\left(p_{l}\right)$, and

iii) Failures are independent and identically distributed.

\section{b. Antipode reliability}

Since the number of antipodes is more than one (for $n>3$ ), the antipodal communication we are concerned about is the communication between $u$ and its unique basic antipode (assuming to be $I$ ). Due to the symmetry in star network, similar analysis can be extended to the communication between the source node and other antipodes. The basic antipode can be reached from the source node by applying the following permutations:

$$
\begin{aligned}
\delta_{\max }= & (1)(23)(45) \ldots(i, i+1) \ldots(n-1, n), \quad \text { for odd } \mathrm{n} ; \\
\delta_{\max }= & (1)(234)(56) \ldots(i, i+1) \ldots(n-1, n) \\
& (12)(34)(56) \ldots(i, i+1) \ldots(n-1, n), \text { for even } \mathrm{n}
\end{aligned}
$$

As such, the antipodal communication can be done concurrently according to the following sequence:

$$
\begin{aligned}
& 2-3-2 \ldots i-(i+1)-i \ldots(n-1)-n-(n-1), \quad \text { for odd } n \\
& 2-3-4-2 \ldots i-(i+1)-i \ldots(n-1)-n-(n-1) \text { or } \\
& 2-3-4-3 \ldots i-(i+1)-i \ldots(n-1)-n-(n-1), \text { for even } n .
\end{aligned}
$$

To have at least one operational optimal path between $u$ and $I$, two different scenarios need to be considered.

Scenario 1. When $n$ is odd, there are a total of $n-1$ disjoint paths of the shortest length of $d_{n}$ between $u$ and $I$, $\left(\pi(1)=1, m=n-1, c=(n-1) / 2, r=c+m=\lfloor 3(n-1) / 2\rfloor=d_{n}\right)$.

Paths distributions can be verified by the following example. For example, there are 6 node-disjoint paths between $u$ and $I$ when $n=7$. Total six disjoint paths can be formulated based on the following permutations:

$$
\begin{aligned}
& \text { (1) } 2-3-2-4-5-4-6-7-6 \\
& \text { (2) } 3-2-4-5-4-6-7-6-3 \\
& \text { (3) } 4-5-4-6-7-6-2-3-2 \\
& \text { (4) } 5-4-6-7-6-2-3-2-5 \\
& \text { (5) } 6-7-6-4-5-4-2-3-2 \\
& \text { (6) } 7-6-4-5-4-2-3-2-7
\end{aligned}
$$

A Boolean technique for the reliability evaluation starts with a sum of products expression for min-paths or cutsets and converts it into an equivalent sum of disjoint products (SDP) expression [9]. In the SDP form, an UP or success (DOWN or failure) state of a node is replaced by its reliability $p_{n}$ or $\left(1-p_{n}\right)$, and the Boolean sum (product) by the arithmetic sum (product). In other words, the SDP expression is interpreted directly as an equivalent probability expression of symbolic reliability. Let $P_{1}, P_{2}, \ldots$ , $P_{h}$ be all $r$-paths between $u$ and $I$, (note that $h=m-l$ ). Then the SDP expression is obtained as follows:

$$
P_{1}+P_{2} \bar{P}_{1}+\ldots+P_{h} \bar{P}_{1} \ldots \bar{P}_{h-1}
$$

where $\bar{P}_{j}$, denotes DOWN events of path $P_{j}$. The probability of UP (operational) for the $i^{\text {th }}$ term $P_{i} \bar{P}_{1} \ldots \bar{P}_{i-1}$ can be evaluated using the conditional probability and the standard Boolean operations. It has been shown that the reliability evaluation for star networks with non-disjoint paths is NP-hard [4]. Clearly, for the same reason, the determination of $D R$ is also intractable. Thus, we attempt to do the next best thing, i.e. derive bounds on $D R$ of the nodedisjoint paths.

Lower Bound: A lower bound on $D R$ can be obtained by considering only the set of $n-1$ node-disjoint paths between $u$ and $I$ with the distance $r$.

$$
D R_{r} \geq 1-\left(1-p_{n}^{r-1}\right)^{n-1}
$$

Above expression uses the principles for a simple parallel reliability block diagram. Note that the bound is quite pessimistic; even for small size star networks, it renders a 
large deviation. Next section presents a tight lower bound. Consider two nodes $u$ and $I$, i.e. $r=3$ when $n$ is 3 . There are only two node-disjoint 3-paths (Fig. 4-a). The expression for $D R_{3}$ in terms of path labels can be attributed to the following:

$$
D R_{3}=1-\left(1-p_{n}^{2}\right)^{2}
$$

When $n=5$, there are two hexagons between $u$ and $I$ having four node-disjoint paths. The corresponding $D R$ can be obtained as follows shown in Fig. 4-b:

$$
D R_{6}=1-\left\{1-P_{n}^{1}\left[1-\left(1-P_{n}^{2}\right)^{2}\right]^{2}\right\}^{2}
$$

Similarly, extending the above concept, the following equation holds for $D R_{r}$ :

$$
\begin{aligned}
& D R_{r}=1-\left\{1-P_{n}^{c-1}\left[1-\left(1-P_{n}^{2}\right)^{2}\right]^{c}\right\}^{c} \\
& =1-\left\{1-P_{n}^{\frac{r-3}{3}}\left[1-\left(1-P_{n}^{2}\right)^{2}\right]^{\frac{r}{3}}\right\}^{\frac{r}{3}}, \text { where } r=3,6,9 \cdots \frac{3(n-1)}{2}
\end{aligned}
$$
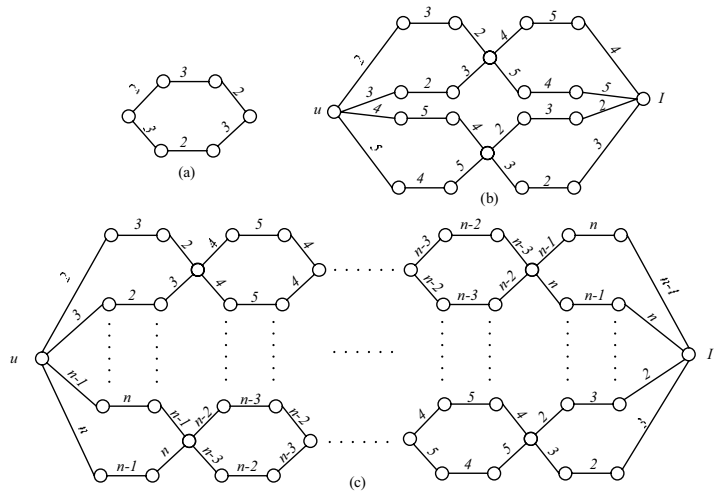

Fig. 4. Hexagon construction of node-disjoint paths between $u$ and its basic antipode $I$.

Scenario 2. When $n$ is even, there are two possibilities: either $\pi(1)=1$ or $\pi(1) \neq 1$, which has been explained in Section 2.3. Paths distribution will be similar to Scenario 1. The lower bound for $D R$ can be derived similarly.

\section{Link failure model}

This section analyzes $D R$ under the link failure model with at most $|F|$ failures, where $F$ represents the set of faulty links. Nodes are assumed to be perfect under this model. As with the node failures, the interest is in the system configuration that has at least one operational optimal path between $u$ and $I$ at the presence of link failures. Since $D R$ analysis under this model is the same as Section 3 except the antipodal communicators, detailed analysis process is not given here for brevity. For the antipodal communications, path distributions will be the same as Section 3.3. The lower bound on $D R$ for the antipodal communication under this model is given as follows:

$D R_{r}=1-\left\{1-\left[1-\left(1-P_{l}^{3}\right)^{2}\right]^{\frac{r}{3}}\right\}^{\frac{r}{3}}$, where $r=3,6,9 \cdots \frac{3(n-1)}{2}$
Above expressions are only for the case where $n$ is odd. Similar lower bound on $D R_{r}$ can be derived where $n$ is even.

\section{Combined node and link failure model}

In the previous two sections, we only consider either nodes or links could fail. However, all network components (nodes and links) can fail in reality. A combined failure model is developed to analyze $D R$ under the case with at most $|F|$ failures, where $F$ represents the sum of faulty nodes and links. As with node failures, the interest is in the system that has at least one operational optimal path between $u$ and $I$ at the presence of node/link failures.

\subsection{Case I $\pi(1)=1$}

There are $m$ optimal node-disjoint paths of the shortest distance $c+m$, and $n-m-1$ non-optimal paths of distance $c+m+2$ between $u$ and $I(m<n, c \leq\lfloor(n-1) / 2\rfloor$, and $r=c+m)$. Total distinct nodes and links in this container is $k=m(2 r-1)+2$.

There will be at least one operational path between $u$ and $I$ if $|F|<m$. Further, since nodes and links are equally likely to fail, it is possible to have one operational path between $u$ and $I$ even when $|F|<2 m-1$.

Now we investigate $D R$ when $|F|=2 m-2$. The conditional probability associated with this event is:

$$
\operatorname{Pr}\left(\text { all r-paths destroyed }|| F|=2 m-2|=2^{2 m-1} /\left(\begin{array}{c}
k-2 \\
2 m-2
\end{array}\right)\right.
$$

Table 3. Prob. of destroying all $r$-paths.

\begin{tabular}{|c|c|c|c|c|c|}
\hline$n$ & $\begin{array}{c}\max . \\
m\end{array}$ & $\begin{array}{c}\max . \\
c\end{array}$ & $r$ & $\begin{array}{c}\text { \# of failures } \\
\text { in container }\end{array}$ & $\begin{array}{c}\text { Prob. having at } \\
\text { least one } \\
\text { optimal path }\end{array}$ \\
\hline 4 & 3 & 1 & 5 & 21 & 0.994653300 \\
\hline 5 & 4 & 2 & 7 & 44 & 0.999981867 \\
\hline 6 & 5 & 2 & 8 & 65 & 0.999999899 \\
\hline 7 & 6 & 3 & 10 & 102 & 1.000000000 \\
\hline 8 & 7 & 3 & 11 & 133 & 1.000000000 \\
\hline
\end{tabular}

\begin{tabular}{|c|c|c|c|c|c|}
\hline$n$ & $\begin{array}{c}\max . \\
m\end{array}$ & $\begin{array}{c}\text { max. } \\
c\end{array}$ & $r$ & $\begin{array}{c}\# \text { of failures } \\
\text { in container }\end{array}$ & $\begin{array}{c}\text { Prob. having at } \\
\text { least one optimal } \\
\text { path }\end{array}$ \\
\hline 4 & 4 & 2 & 4 & 10 & 0.911111111 \\
\hline 5 & 5 & 2 & 5 & 12 & 0.939393939 \\
\hline 6 & 6 & 3 & 7 & 24 & 0.996047431 \\
\hline 7 & 7 & 3 & 8 & 27 & 0.997264957 \\
\hline 8 & 8 & 4 & 10 & 44 & 0.999882137 \\
\hline 9 & 9 & 4 & 11 & 48 & 0.999917772 \\
\hline
\end{tabular}

Table 4. Prob. of destroying all $r$-paths.

Above equation accounts for $2^{2 m-1}$ mutually-exclusive distributions destroying all $m$ optimal paths. Subtraction of the probability of occurrence of these events from 1 will 
naturally give probability of having at least one operational $r$-path in the container and thus the $D R$. In reality the likelihood of having all $r$-paths failed due to the node and link failures is very small. The probability of existing at least one optimal path when $|F|=2 m-2$ are presented in Table 3. Results in Fig. 4 show that chance of existing one optimal path at the presence of node/link failures can be assured when the shortest distance is larger than 10 .

\subsection{Case II: $\pi(1) \neq 1$}

There will be at least one operational path between $u$ and $I$ if $|F|<c$. Further, since nodes and links are equally likely to fail, it is possible to have one operational path between two given node $u$ and $I$ even when $|F|<2 c-1$. Now we investigate $D R$ when $|F|=2 c-2$. The conditional probability associated with this event is: $\operatorname{Pr}$ (all r-paths destroyed ||$F|=2 m-2|=2^{2 m-1} /\left(\begin{array}{c}k-2 \\ 2 m-2\end{array}\right)$

Above equation accounts for the $2^{2 c-1}$ mutually-exclusive distributions that destroy all $c$ optimal paths. The probability of existing at least one optimal path when $|F|=$ $2 c-2$ are presented in Table 4. Compared with Fig. 3, DR under this model shown in Fig. 5 demonstrates higher robustness of the star network. Even in the low shortest distance region, star networks under the combined node and link failure model have better tolerance to failures than the cases where either node or link will fail. .

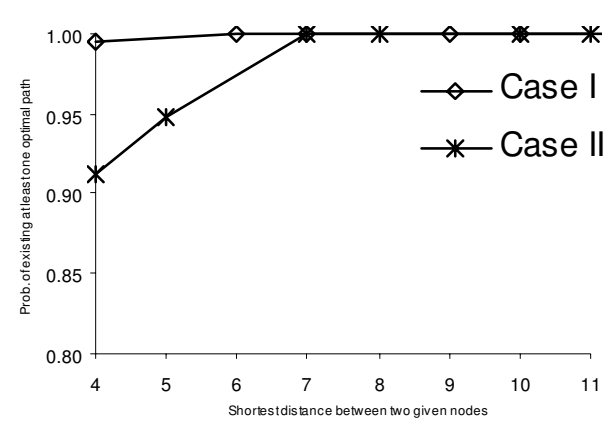

Fig. 4. Prob. of existence of at least one optimal path.

\subsection{Special case: antipodal reliability}

Antipode reliability under this model can be analyzed similar to the node failure mode described in Section 3.3. The lower bound on $D R$ of the antipodal communication (only for odd $n$ case) is derived as follows by using the same analysis scheme in Section 3.3:

$$
D R_{r}=1-\left\{1-P_{n}^{\frac{r-3}{3}}\left[1-\left(1-P_{n}^{2} P_{l}^{3}\right)^{2}\right]^{\frac{r}{3}}\right\}^{\frac{r}{3}}, \text { where } r=3,6,9 \cdots \frac{3(n-1)}{2}
$$

\section{Conclusion}

A figure of merit called distance reliability has been introduced for the reliability analysis of star interconnection networks. This measure is appealing for the robust networks (such as star network) since it poses stringent requirements on the connection of two nodes; i.e. not only do two nodes have to be connected, but the distance between them must be the shortest. We presented a deterministic formulation of distance reliability when the number of faults is bounded using the combinatorial method. For each of the node, link and node/link failure model, two different cases depending on the relative positions of the source $\&$ destination, are analyzed to compute $D R$. The antipodal reliability is also considered as a special case to further demonstrate the fault tolerance of star networks.

\section{References}

[1] S. B. Akers and B. Krishnamurthy, "A group-theoretic model for symmetric interconnection networks," in Proc. International Conference on Parallel Processing, 1986, pp. 216-223.

[2] S. B. Akers, D. Horel, and B. Krishnamurthy, "The star network: An attractive alternative to the n-cube," in Proc. International Conference on Parallel Processing, 1987, pp. 393-400.

[3] N. Bagherzadeh, M. Dowd, and N. Nassif, "Embedding an arbitrary binary tree into the star graph," IEEE Transactions on Computers, vol. 45, no. 4, April 1996, pp. 475-481.

[4] M. S. Chang, D. J. Chen, M. S. Lin, and K. L. Ku, "The distributed program reliability analysis on star topologies," in Proc. 1998 International Conference on Parallel and Distributed Systems, 1998, pp.100-106.

[5] K. Day and A. Tripathi, "A comparative study of topologies properties of hypercube and star networks," IEEE Transactions on Parallel and Distributed Systems, vol. 5, no. 1, Jan. 1994, pp. 31-38.

[6] N. Imani, H. S. Azad, and S. G. Akl, "On some combinatorial properties of star graph," in Proc. $8^{\text {th }}$ International Symposium on Parallel Architectures, Algorithms and Networks, 2005, pp. 58-65.

[7] A. E. Kiasari and H. Sarbazi-Azad, "A comparative performance analysis of n-cubes and star networks," in Proc. $20^{\text {th }}$ International Parallel and Distributed Processing Symposium, 2006, pp. 25-29.

[8] S. Latifi, "On the fault-diameter of the star network," Information Processing Letters, vol. 46, no. 3, June 1993, pp. 143-150.

[9] S. Latifi, "Parallel dimension permutations on star network," IFIP Trans. A: Comp. Sci. Tech., A23, 1993, pp. 191-201.

[10] N. Nassif and N. Bagherzadeh, "A grid embedding into the star network for image analysis solutions," Information Processing Letters, vol. 60, no. 5, Dec. 1996, pp. 255-260.

[11] S. Sur and P. K. Srimani, "Topological properties of star networks," Computers \& Mathematics with Applications, vol. 25, no. 12, 1993, pp. 87-98. 